\title{
Einstein's Rocket Ship, the Deflection of Light and the Precession of the Orbital Perihelion
}

\author{
Firmin J. Oliveira, MS \\ Software Engineer (retired), East Asian Observatory/ \\ James Clerk Maxwell Telescope, Hilo, Hawaii, USA
}

Doi: 10.19044/esj.2018.v14n15p535 URL:http://dx.doi.org/10.19044/esj.2018.v14n15p535

\begin{abstract}
The nature of the principle of equivalence is explored. The light ray travel path in an accelerated reference frame, a rocket ship, is described and the rocket ship model is used to derive the deflection of light by a massive body. By accounting for the effect of the velocity of the accelerated observer relative to an inertial frame, the additional deflection angle is obtained due to the aberration of the light beam. This model is applied to the deflection of light by a central gravitational field, giving the total deflection angle in agreement with the standard result. Also, a novel approach is given by considering the deflection of light by a massive body to obtain the precession of the perihelion of a planet.
\end{abstract}

Keywords: Equivalence principle, Light deflection, Perihelion advance

\section{Introduction}

The deflection by the Sun of light from a distant star is explored within the principle of equivalence. An accelerated system, a constantly accelerating rocket ship, is setup equivalent to the gravitational acceleration of the Sun. The path of a beam of light is analyzed. We show that including the effect of light aberration due to motion of the observer, the deflection in the rocket ship can duplicate the deflection of light passing through the gravitational field of the sun. Then, by taking into account the aberration of light, the problem of the light beam grazing the Sun is resolved and the standard value is obtained. We also consider the advance of the perihelion of a planetary orbit by the deflection of light relative to the orbit, deriving the standard formula.

\section{Lorentz and Rindler transformations}

A rocket ship accelerates at a uniform rate a relative to a stationary inertial system $\mathrm{O}^{\prime}$ having coordinates $\mathrm{x}^{\prime}, \mathrm{y}^{\prime}, \mathrm{z}^{\prime}, \mathrm{t}^{\prime}$. The acceleration is along the $\mathrm{x}^{\prime}$ axis of $\mathrm{O}^{\prime}$. Let $\mathrm{O}$ be the accelerating coordinate system for the rocket ship 
having coordinates $\mathrm{x}, \mathrm{y}, \mathrm{z}, \mathrm{t}$. Assume the axes $\mathrm{x}^{\prime}, \mathrm{y}^{\prime}, \mathrm{z}^{\prime}$ and $\mathrm{x}, \mathrm{y}, \mathrm{z}$ are parallel, respectively between $\mathrm{O}^{\prime}$ and $\mathrm{O}$ and that the times $\mathrm{t}^{\prime}$ and $\mathrm{t}$ are synchronized at $\mathrm{t}^{\prime}$ $=\mathrm{t}=0$ when their origins intersect at zero velocity.

We use the Lorentz equations for transformations between inertial frames $\mathrm{O}^{\prime}$ and $\mathrm{O}^{\prime \prime}$ having coordinates $\mathrm{x}^{\prime \prime}, \mathrm{y}^{\prime \prime}, \mathrm{z}^{\prime \prime}, \mathrm{t}^{\prime \prime}$ with spatial coordinates respectively parallel between $\mathrm{O}^{\prime}$ and $\mathrm{O}^{\prime \prime}$ and clocks synchronized at $\mathrm{t}^{\prime}=\mathrm{t}^{\prime \prime}=$ 0 , moving at relative velocity $\mathrm{v}$, the transformation equations given by

$$
\begin{aligned}
& x^{\prime \prime}=\mathrm{Y}^{\prime}\left(x^{\prime}-v t^{\prime}\right), \\
& c t^{\prime \prime}=\mathrm{Y}^{\prime}\left(c t^{\prime}-\frac{v x^{\prime}}{c}\right), \\
& y^{\prime \prime}=y^{\prime}, \\
& z^{\prime \prime}=z^{\prime},
\end{aligned}
$$

where

$$
\mathrm{Y}^{\prime}=\frac{1}{\sqrt{1-v^{2} / c^{2}}}
$$

The inverse Lorentz transformation equations are given by

$$
\begin{aligned}
& x^{\prime}=\mathrm{Y}^{\prime}\left(x^{\prime \prime}+v t^{\prime \prime}\right), \\
& c t^{\prime}=\mathrm{Y}^{\prime}\left(c t^{\prime \prime}+\frac{v x^{\prime \prime}}{c}\right), \\
& y^{\prime}=y^{\prime \prime}, \\
& z^{\prime}=z^{\prime \prime} .
\end{aligned}
$$

We will also use the Rindler coordinate system (Knorr, 2010) to transform between stationary inertial frame $\mathrm{O}^{\prime}$ and frame $\mathrm{O}$ having constant acceleration a with respect to $\mathrm{O}^{\prime}$, given by

$$
\begin{aligned}
& x=\sqrt{\left(x^{\prime}+\frac{c^{2}}{a}\right)^{2}-c^{2} t^{\prime 2}}-\frac{c^{2}}{a}, \\
& c t=\frac{c^{2}}{a} \operatorname{artanh}\left(\frac{c t^{\prime}}{x^{\prime}+c^{2} / a}\right), \\
& y=y^{\prime}, \\
& z=z^{\prime} .
\end{aligned}
$$


The inverse transform, from $\mathrm{O}$ to $\mathrm{O}$ ' is given by

$$
\begin{aligned}
& x^{\prime}=\left(x+\frac{c^{2}}{a}\right) \cosh \left(\frac{a t}{c}\right)-\frac{c^{2}}{a}, \\
& c t^{\prime}=\left(x+\frac{c^{2}}{a}\right) \sinh \left(\frac{a t}{c}\right), \\
& y^{\prime}=y, \\
& z^{\prime}=z .
\end{aligned}
$$

In the stationary inertial frame $\mathrm{O}^{\prime}$, the trajectory of a fixed point in $\mathrm{O}$ is seen as a hyperbola given by

$$
x^{\prime 2}+y^{\prime 2}+z^{\prime 2}-c^{2} t^{\prime 2}=\left(x+\frac{c^{2}}{a}\right)^{2}+y^{2}+z^{2}
$$

where $\mathrm{x}, \mathrm{y}$ and $\mathrm{z}$ are the coordinates of the fixed point in the accelerated frame. The velocity $v$ of the rocket ship frame $\mathrm{O}$ relative to $\mathrm{O}^{\prime}$ at time $\mathrm{t}^{\prime}$ is given by,

$$
v=\frac{a t^{\prime}}{\sqrt{1+a^{2} t^{\prime 2} / c^{2}}},
$$

where $\mathrm{c}$ is the speed of light in vacuum and the rocket ship has a velocity $\mathrm{v}=0$ at $\mathrm{t}^{\prime}=0$.

\section{Propagation of light in an accelerated system}

In a rocket ship $\mathrm{O}$, a light pulse generator $\mathrm{A}$ is on a pedestal at height $\mathrm{x}=\mathrm{k}$ and position $\mathrm{y}=\mathrm{d}$.

At position $\mathrm{y}=0$, there is a

vertical assembly of light pulse detectors $A_{L}$ extending in the $\mathrm{x}$ direction from $\mathrm{x}=0$ to height $\mathrm{x}=\mathrm{k}$,

as shown in Figure 1. Assume that the clocks in $\mathrm{O}$ and $\mathrm{O}^{\prime}$ are all set to $\mathrm{t}=\mathrm{t}^{\prime}=$ 0 when the velocity of the

rocket ship is $\mathrm{v}=0$ with respect to $\mathrm{O}^{\prime}$. Assume that at time

$$
t=t_{1}=0
$$

on $\mathrm{A}$ 's clock in $\mathrm{O}$, which corresponds to $\mathrm{t}^{\prime}=\mathrm{t}^{\prime}{ }_{1}=0$ on the clock at the origin of $\mathrm{O}^{\prime}$, a pulse of light 
is emitted from $\mathrm{A}$ at an angle of $\mathrm{p}_{0}$ with respect to the vertical $\mathrm{x}$ direction and is directed toward the vertical assembly of detectors at $\mathrm{y}=0$. The instantaneous velocity of the rocket with respect to $\mathrm{O}^{\prime}$ at time $\mathrm{t}^{\prime}{ }_{1}=0$ is

$$
v=v_{1}=0
$$

by (19). The light pulse is received on detector $A_{L}$ at height $x$ at time $t_{2}$ on $A_{L}$ 's clock at a vertical distance $h$ below and $d$ to the left of the emitter at $A$. The distance $\mathrm{d}$, the lateral dimension of the rocket ship perpendicular to the direction of acceleration. At the time $t_{2}$, the time in $\mathrm{O}^{\prime}$ is $\mathrm{t}_{2}^{\prime}$ and the instantaneous velocity of the rocket is given by (19),

$$
v_{2}=\frac{a t^{\prime}{ }_{2}}{\sqrt{1+a^{2} t^{\prime 2} / c^{2}}} \text {. }
$$

By the Lorentz transformation, the clock at $A_{L}$ of coordinate system $\mathrm{O}$ which is moving instantaneously at velocity $\mathrm{v}_{2}$ with respect to $\mathrm{O}^{\prime}$, as observed in the inertial system $\mathrm{O}^{\prime \prime}$ which is moving at constant velocity $\mathrm{v}_{2}$ with respect to $\mathrm{O}^{\prime}$ and by which the accelerated system $\mathrm{O}$ is instantaneously at rest, the time in $\mathrm{O}$ " and thus $\mathrm{O}$ is given by

$$
t_{2}=t{ }_{2}^{\prime \prime}=\mathrm{Y} t^{\prime}{ }_{2} \text {, }
$$

where $\gamma$ is given by (5),

$$
\mathrm{Y}=\frac{1}{\sqrt{1-v_{2}^{2} / c^{2}}}
$$

Relative to the rocket ship frame, which has a velocity $\mathrm{v}\left(\mathrm{t}^{\prime}\right)$ given by (19), the light ray falls a distance $\mathrm{h}$ in $\mathrm{O}$ as observed in frame $\mathrm{O}$ " moving at velocity $\mathrm{v}_{2}$ with respect to $\mathrm{O}^{\prime}$ so that $\mathrm{O}$ is seen to be momentarily at rest, is given by

$$
h=\int_{0}^{t_{2}} v(u) d u=\frac{c^{2}}{a} \int_{0}^{t_{2}} \frac{a u / c}{\sqrt{1+(a u / c)^{2}}} d u=\frac{\sqrt{1+\left(a t_{2} / c\right)^{2}}-1}{a / c^{2}},
$$

where the coordinates of $\mathrm{O}$ and $\mathrm{O}^{\prime \prime}$ are $\mathrm{x}=\mathrm{x} ", \mathrm{t}=\mathrm{t}$ ", $\mathrm{y}=\mathrm{y}^{\prime \prime}$ and $\mathrm{z}=\mathrm{z}^{\prime \prime}$.

Substituting from (23), $\mathrm{t}_{2}=\gamma \mathrm{t}_{2}$ into (25) and assuming that a $\gamma \mathrm{t}_{2}^{\prime}<<$ yields 


$$
h=\frac{\sqrt{1+\left(a \bigvee t^{\prime}{ }_{2} / c\right)^{2}}-1}{a / c^{2}} \approx \frac{a \mathrm{Y}^{2} t_{2}{ }_{2}}{2} .
$$

From Figure 1., assuming propagation approximately in a straight line, the distance $\mathrm{g}$ traveled by the light pulse between emission at $\mathrm{A}$ and detection at $\mathrm{A}_{\mathrm{L}}$ is given by Pythagoras' theorem, $g^{2}=d^{2}+h^{2}$, which can be written as

$$
g=d \sqrt{1+\frac{h^{2}}{d^{2}}}
$$

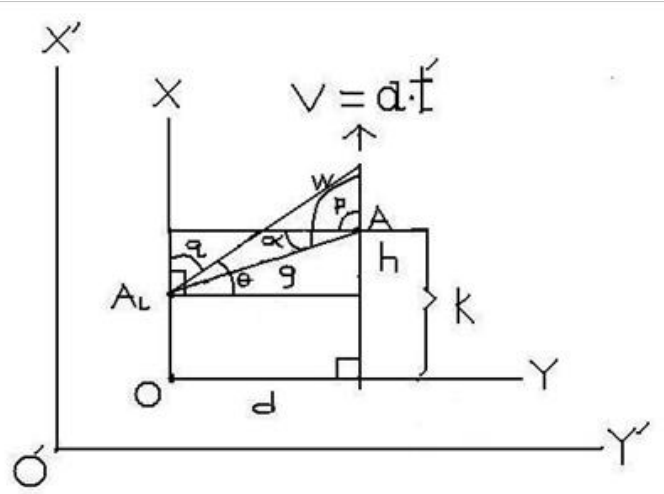

Figure 1. Rocket Ship in frame $\mathrm{O}$ accelerating at rate a with respect to inertial frame $\mathrm{O}^{\prime}$.

Since the initial times $\mathrm{t}_{1}{ }_{1}=\mathrm{t}_{1}=0$, the time interval $\mathrm{t}_{2}$ of travel of the light ray in $\mathrm{O}$ is given by (23), where the time $\mathrm{t}_{2}{ }_{2}$ in inertial frame $\mathrm{O}^{\prime}$ for the light pulse to travel in a straight line the distance $d$ in vacuum is given by

$$
t_{2}^{\prime}=\frac{d}{c}
$$

Substituting (28) into (27), $\mathrm{g}$ is given by

$$
g \approx d \sqrt{1+\left(\frac{\mathrm{Y}^{2} a d}{2 c^{2}}\right)^{2}} \approx d\left(1+\frac{\mathrm{Y}^{4} a^{2} d^{2}}{8 c^{4}}\right) .
$$

\section{Deflection of light in the accelerated system}

From Figure 1., the light beam is incident at the reception point $A_{L}$ at an angle $\alpha$ given by 


$$
\tan (\alpha)=\frac{h}{d} \approx \frac{a \mathrm{Y}^{2} t_{2}^{\prime 2}}{2 d}=\frac{\mathrm{Y}^{2} a d}{2 c^{2}},
$$

which yields

$$
\alpha \approx \arctan \left(\frac{\mathrm{Y}^{2} a d}{2 c^{2}}\right) .
$$

Now, because the detector at point $A_{L}$ in frame $\mathrm{O}$ has a velocity $\mathrm{v}_{2}$ with respect to stationary inertial system $\mathrm{O}^{\prime}$ at the time of reception of the light pulse, then relative to inertial frame $\mathrm{O}^{\prime \prime}$ also moving at velocity $\mathrm{v}_{2}$ with respect to $\mathrm{O}^{\prime}$ in which accelerated system $\mathrm{O}$ is temporarily at rest when the light pulse arrives, the apparent angle $\mathrm{q}$ of the incoming light pulse with the vertical $\mathrm{x}$ axis as seen by the observer in $\mathrm{O}^{\prime \prime}$ is determined by the aberration of light (Bergmann, 1976), which is given by

$$
\tan (q)=\frac{\sin (w)}{\mathrm{V}\left(\cos (w)-v_{2} / c\right)}
$$

where

$$
w=p-\alpha
$$

and $\mathrm{p}$ (see Figure 1.) is the initial angle of transmission of the signal. The observed angle of deflection $\theta$ is given by

$$
\theta=\pi / 2-q
$$

This implies that

$$
\tan (\theta)=\tan (\pi / 2-q)=\frac{1}{\tan (q)}
$$

From (32) this gives

$$
\tan (\theta)=\frac{Y\left(\cos (w)-v_{2} / c\right)}{\sin (w)} .
$$

Deflection of light in the accelerated system calibrated to a massive body 
The deflection of a ray of light incident on a massive body such as a star is well known (Einstein, 1952), with the total angle of deflection given by $\theta_{\mathrm{E}}=4 \mathrm{G} \mathrm{M} / \mathrm{R}^{2}$, where $\mathrm{G}$ is Newton's gravitation constant, $M$ is the mass of the star and $\mathrm{R}$ is the impact parameter, the closest distance of approach of the incident light ray to the center of the star. Our aim is to obtain this deflection amount to first order in $\mathrm{v} / \mathrm{c}$ in the rocket ship analogy.

To this end, we apply the concept of the equivalence of an inertially accelerating system to the gravitational system of the star. We anticipate this will not be symmetrical in the sense that a star has a centrally varying field where as the rocket ship has a constant field. From the rocket ship model of the previous section we make the following definitions. Assume that the acceleration of the rocket ship is

$$
a=\frac{G M}{R^{2}},
$$

and that the width of the rocket ship has the value

$$
d=\frac{8 R}{3} .
$$

From (28) and (38) we get

$$
t^{\prime}{ }_{2}=\frac{d}{c}=\frac{8 R}{3 c} .
$$

Then from (22), (37) and (39) the velocity of the rocket ship frame $\mathrm{O}$ at the time of detection $t_{2}$ of the light pulse at $A_{L}$ is given by

$$
v_{2} \approx \frac{8 G M}{3 c R} \text {. }
$$

Setting the initial transmission angle to $p=-\pi / 2$, we have from (31) and (33)

$$
w=-\pi / 2-\alpha,
$$

where

$$
\alpha \approx \arctan \left(\frac{4 \gamma^{2} G M}{3 c^{2} R}\right) .
$$


The angle of deflection is $\theta$. Using (36) with (41) gives us

$$
\tan (\theta)=\frac{\mathrm{Y}\left[\cos (-\pi / 2-\alpha)-v_{2} / c\right]}{\sin (-\pi / 2-\alpha)}=\frac{\mathrm{Y}\left[\sin (\alpha)+v_{2} / c\right]}{\cos (\alpha)}
$$

Substituting for $\mathrm{v}_{2}$ and $\alpha$ from (40) and (42) into (43) we obtain

$$
\tan (\theta) \approx \frac{Y\left\{\sin \left[\arctan \left(\mathrm{V}^{2} \beta / 2\right)\right]+\beta\right\}}{\cos \left[\arctan \left(\mathrm{Y}^{2} \beta / 2\right)\right]},
$$

where

$$
\beta=\frac{v_{2}}{c} \approx \frac{8 G M}{3 c^{2} R} .
$$

For the Sun, (4/3) G M / $\mathrm{c}^{2} \mathrm{R} \approx 3 \times 10^{-6}$ and $\gamma \approx 1$, so we can make a first order approximation to (44) which gives us

$$
\theta \approx \mathrm{V}\left(\mathrm{V}^{2} / 2+1\right) \beta \approx \frac{4 G M}{c^{2} R},
$$

which equals the standard value $\theta_{\mathrm{E}}$ given above. For the rocket ship frame $\mathrm{O}$ being equivalent to the Solar gravitational system we have acceleration $\mathrm{a}=$ $2.741 \times 10^{4} \mathrm{~cm} \mathrm{~s}^{-2}$, width $\mathrm{d}=1.856 \times 10^{11} \mathrm{~cm}$, total time $\mathrm{t}_{2}{ }_{2}=6.191 \mathrm{~s}$, and velocity at detection time $\mathrm{v}_{2}=1.697 \times 10^{5} \mathrm{~cm} \mathrm{~s}^{-1}$. Also, $\beta=\mathrm{v}_{2} / \mathrm{c}=5.661 \mathrm{x}$ $10^{-6}$ and $\gamma=1$ to ten decimal places. For the total deflection (46) we get angle $\theta=8.491 \times 10^{-6} \mathrm{rad} .=1.751$ arcsec .

\section{The deflection of light by a central gravitational field}

By the equivalence principle, we should be able to apply the accelerated frame analogy of the previous section to the real case of a centrally directed gravitational field such as a massive star. The light ray from a distant source is deflected radially toward the center of the star as it passes by, acquiring a velocity perpendicular to its original line of approach. Construct an xy coordinate system originating at the center of the star, with the light ray traveling from the negative to positive direction and initially parallel to the $y$ axis (Wortzman, 2014). Let the velocity of the light ray be $\mathbf{v}=\mathrm{v}_{\mathrm{x}} \hat{\mathbf{e}}_{\mathbf{x}}+\mathrm{v}_{\mathrm{y}} \hat{\mathbf{e}}_{\mathbf{y}}$ where $\hat{\mathbf{e}}_{\mathbf{x}}$ and $\hat{\mathbf{e}}_{\mathbf{y}}$ are unit vectors along the $\mathrm{x}$ and $\mathrm{y}$ directions respectively, and $\mathrm{v}_{\mathrm{x}}=0$ and $\mathrm{v}_{\mathrm{y}}=\mathrm{c}$ initially. The $\mathrm{x}$ axis of the coordinate system intersects the 
point of closest approach of the light ray at $\mathrm{x}=\mathrm{R}$. The radial distance $\mathrm{r}$ of the photon from the center of the coordinate system makes the angle $\varphi$ with the positive $\mathrm{x}$ axis.

The acceleration $\mathrm{a}_{\mathrm{x}}$ in the $\hat{\mathbf{e}}_{\mathbf{x}}$ direction due to the star's gravity upon the photon at position $r=\sqrt{y^{2}+R^{2}}$ is given by

$$
a_{x}=\frac{-G M \cos (\phi)}{y^{2}+R^{2}}
$$

where $\cos (\phi)=R / \sqrt{y^{2}+R^{2}}$. The velocity $\mathrm{dv}_{\mathrm{x}}$ in the $\hat{\mathbf{e}}_{\mathrm{x}}$ direction is given by $\mathrm{dv}_{\mathrm{x}}=\mathrm{a}_{\mathrm{x}} \mathrm{dt}=\mathrm{a}_{\mathrm{x}} \mathrm{dy} / \mathrm{c}$, where dy / dt $=\mathrm{c}$ is the velocity of light in the $\hat{\mathbf{e}}_{\mathrm{y}}$ direction. Then the velocity $\mathrm{v}_{\mathrm{x}}$ is obtained by integrating (47) over all $\mathrm{y}$,

$$
v_{x}=\int_{-\infty}^{\infty} \frac{-G M R}{\left(y^{2}+R^{2}\right)^{3 / 2}} \frac{d y}{c}=\frac{-2 G M}{c R} .
$$

In this case, from (30), $\tan (\alpha)=\Delta \mathrm{x} / \Delta \mathrm{y}=\left(\mathrm{v}_{\mathrm{x}} \Delta \mathrm{t}\right) /\left(\mathrm{v}_{\mathrm{y}} \Delta \mathrm{t}\right)=\mathrm{v}_{\mathrm{x}} / \mathrm{v}_{\mathrm{y}}$, for some suitable time interval $\Delta \mathrm{t}$.

With $v_{y}=c$, we have from (48) the angle of deflection $\alpha$ given by

$$
\tan (\alpha)=\frac{v_{x}}{v_{y}}=\frac{-2 G M}{c^{2} R}
$$

But we know $\alpha$ is only half the deflection angle ( by $\theta_{\mathrm{E}}$ above.) However, from our experience with Einstein's rocket ship, we realize that the light beam is moving at a velocity $v_{x}$ in the $\hat{\mathbf{e}}_{\mathbf{x}}$ direction, which means that there will be an aberration effect when the light ray is observed at a large distance y from the star. Then the total deflection of the light beam is given by (43)

with $\gamma=1$ and $\mathrm{v}_{2}=-\mathrm{v}_{\mathrm{x}}$,

$$
\tan (\theta)=\frac{\sin (\alpha)+v_{x} / c}{\cos (\alpha)} \approx \frac{2 v_{x}}{c},
$$

where, since $\alpha$ is small, $\sin (\alpha) \approx \alpha=\mathrm{v}_{\mathrm{x}} / \mathrm{c}$ and $\cos (\alpha) \approx 1$. And, since $\tan (\theta) \approx \theta$ for small $\theta$, substituting from (49) into (50) and taking the absolute value, we get the expected result 
$\theta \approx \frac{4 G M}{c^{2} R}$.

\section{Precession of the orbital perihelion}

Assume that a ray of light from a distant source interacts with a star of mass $M$ at a closest distance of $R=4 r / 3$ from the center of the star, where $r$ is the radial coordinate of the elliptical orbit of a satellite around the star. Using the result from the previous section, the deflection of the light ray can be expressed by (51)

$$
\theta=\frac{4 G M}{c^{2}(4 r / 3)}=\frac{3 G M}{c^{2} r}
$$

when observed at a large distance from the star. With $\mathrm{r}=\mathrm{L} /[1+\varepsilon \cos (\varphi)]$ into (52), the deflection of the light ray approaching as close as $R=4 \mathrm{r} / 3$ to the star is given by

$$
\theta=\frac{3 G M}{c^{2}}\left[\frac{1+\epsilon \cos (\phi)}{L}\right]
$$

where $\varphi$ is the angle that $r$ makes with the line through the points of perihelion and aphelion, $\varepsilon$ is the eccentricity of the ellipse and $\mathrm{L}=\mathrm{a}\left(1-\varepsilon^{2}\right)$ is the semilatus rectum with a the semi-major axis. Multiplying (53) by $\mathrm{d} \varphi$ and integrating between 0 and $2 \pi$ gives the total deflection of all the light rays which impact the star at distances of $4 / 3$ of the elliptical orbit, yielding

$$
\delta \psi=\int_{0}^{2 \pi} \theta d \phi=\frac{3 G M}{c^{2} L} \int_{0}^{2 \pi}[1+\epsilon \cos (\phi)] d \phi=\frac{6 \pi G M}{c^{2} L} .
$$

By Kepler's third law, G M $=4 \pi^{2} \mathrm{a}^{3} / \mathrm{T}^{2}$ where $\mathrm{T}$ is the period of the elliptical orbit. Substituting this into the right hand side of (54) yields Einstein's result (Einstein, 1952),

$$
\delta \psi=\frac{24 \pi^{3} a^{2}}{c^{2} T^{2}\left(1-\epsilon^{2}\right)} .
$$

For planet Mercury, with $\mathrm{a} \approx 0.387096 \mathrm{AU}, \mathrm{T} \approx 0.24085 \mathrm{yr}, \varepsilon \approx 0.205622$ and $\mathrm{c} \approx 63245.98646 \mathrm{AU} \mathrm{yr}^{-1},(55)$ gives a perihelion advance of $\delta \Psi \approx 0.4297$ " $\mathrm{yr}^{-1}$, which agrees with the observed result. 


\section{Conclusion}

It is satisfying to know that special relativity and Newtonian mechanics can address light deflection and perihelion precession in a straight forward manner. Aberration seems to play a key role in the interaction of gravity and light. Although we have not shown it, Newtonian mechanics can also explain the gravitational redshift, by attributing mass to light, and even the expansion of the universe, though not the accelerated expansion. And our analysis of light deflection also explains gravitational lensing, another cosmological effect. The three tests for general relativity theory, light deflection, gravitational red shift and precession of Mercury's perihelion, would seem to be already covered by the earlier theories, though it may not have been realized at past times.

\section{References:}

1. Knorr, B. (2010) Uniform relativistic acceleration. http://www.physik.uni-leipzig.de/ schiller/ed10/Uniform relativistic acceleration.pdf

2. Bergmann, P. G. (1976) Introduction to the theory of relativity (p. 37). Dover Publications, Inc., New York

3. Einstein, A. (1952) The foundation of the general theory of relativity (p. 63).

Dover Publications, Inc., New York

4. Wortzman, D. (2014) Bending of starlight by the sun. 